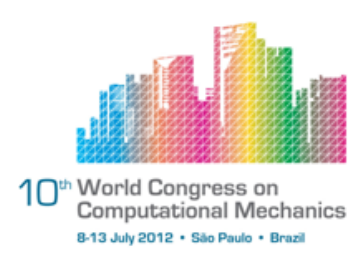

\title{
STUDY OF THE BREATHING MECHANISM OF AN ELLIPTICAL CRACK IN A ROTATING SHAFT WITH AN ECCENTRIC MASS
}

\author{
B. Muñoz-Abella, P. Rubio, L. Montero, L. Rubio \\ Department of Mechanical Engineering, University Carlos III of Madrid. Spain
}

\begin{abstract}
Rotative machinery (blades, rotors, compressors,..) are used quite often in several industries such as aerospace, nuclear, oil, transportation and industry processes in general. Operation failures of this kind of machinery are related with the presence and growing of fatigue cracks in one of the main components: the shafts. The shafts, that perform in bending and torsion, present additionally misalignments or/and unbalances that alter the normal function of the components. The unbalance in a rotating system can be produce by a eccentric mass. When a cracked shaft rotates, the crack opens and closes in what is called the breathing mechanism in which the crack passes from an open state to a close state with a transition between both situations producing a partial opening or closing of the crack. On the other hand it is very frequent to consider cracks with straight fronts, but the experience shows that most of the cracks that appear in rotating shafts are elliptical. In this paper we present the numerical model (Finite Element Method) of the quasi-static behaviour of an unbalance cracked shaft (with elliptical front) considering an eccentric mass. The model chosen for this study is the classical Jeffcott rotor. To simulate the rotation of the shaft, different angular positions have been considered. The influence of the mass eccentricity in the opening of the crack has been studied considering different angles of eccentricity measured from the position of the crack. In this paper we present the comparison of the partially opening/closing of the crack considering different angles of rotation and different positions of the eccentricity. The work allows to know the influence of the unbalance of rotating shafts in the crack breathing mechanism and will allow to predict the influence of this behavior on the values of the Stress Intensity Factor and in the propagation of cracks.
\end{abstract}

Keywords: shaft, elliptical crack, eccentricity, breathing mechanism, rotative machinery.

\section{INTRODUCTION}

The failures of machines are produced quite often by the presence and propagation of fatigue cracks due to the loads and solicitations they carry. Those failures sometimes are catastrophic and produce personal injuries or economic problems. The increasing importance of safety and costs derived from failure in machinery has pushed the researchers in the field of damage detection to analyze the behavior of mechanical components with defects. 
Shafts, that are one of the main components of machines, perform in rotation and in bending and torsion. All of them together, can produce the shaft failure by generation and propagation of fatigue cracks.

In the presence of a crack, when a shaft rotates, the crack opens and closes once per revolution. The opening and closing of the crack has been modeled in different ways. The simplest one is to consider that the crack is open or closed, so that the crack is half the rotation in the open state and the other half in the closed one. This model has been used quite often due to its simplicity. However the most feasible behavior of the crack is that called the breathing behavior. In this case, the crack passes from the closed state to the open state gradually in a rotation. The crack is closed when it is situated in the compression zone of the shaft and it is open when situated in the tensile zone. The transition between both situations produces partial opening or closing of the crack when the static deflection dominates the performance of the rotating shaft. The partial opening/closing of the crack has been studied, numerically or analytically, by different authors [8,5,1] always considering an aligned and balanced shaft. The opening and closing of the crack is very much influenced by the the values taken by the the Stress Intensity Factor (SIF) at the crack front as the shaft rotates. The crack is open while the SIF at the front remains positive, otherwise the crack will be closed.

In the real performance of shafts it is very usual that the shafts present unbalances or misaligments that modify the normal behavior of the components. A very common unbalance of shafts is due to the presence of eccentric masses [14,5,10,3]. The unbalance, as mention before, modifies the dynamic behavior of the rotating shafts and may hide the presence of the cracks or, on the other hand, can increase the effects of the cracks.

Most of the aforementioned studies consider straight front cracks, and are less frequent those analysing elliptical front shapes $[4,2,15,13]$, although this is the type of cracks present in real cracked shafts.

In this paper we present the numerical study of the influence of the eccentricity in a rotating cracked shaft using a finite element model of a cracked Jeffcott rotor. The model includes the presence of an elliptical crack. The analysis has been made using the commercial finite element code ABAQUS. We present the comparison, for each angle of rotation, of the partially opening/closing of the crack for different positions of the eccentricity. The work allows to know the influence of the unbalance of rotating shafts in the crack breathing mechanism and allows to predict the influence of this behavior on the values of the stress intensity factor and in the propagation of elliptical cracks.

\section{THE CRACKED SHAFT MODEL}

The model chosen for this study is the classical Jeffcott rotor widely used in rotordynamics $[11,6,7,9,10,3]$. This simple but useful model consists in a massless shaft simply supported at the ends, with a concentrated mass (a disc). The crack is situated at the midspan of the shaft having an elliptical front oriented on a plane normal to the axis of the shaft (Figure 1). The eccentric mass has been placed on the disc of the Jeffcott rotor as an additional mass as can be seen in Figure 2.The round bar total length is equal to $900 \mathrm{~mm}$, whereas the diameter is $D=20 \mathrm{~mm}$. The material of the shaft is aluminium with the following mechanical properties: Youngs Modulus $E=72 \mathrm{GPa}$, Poisson ratio $\mu=0.33$ and density $\rho=2800 \mathrm{Kg} / \mathrm{m}^{3}$. 


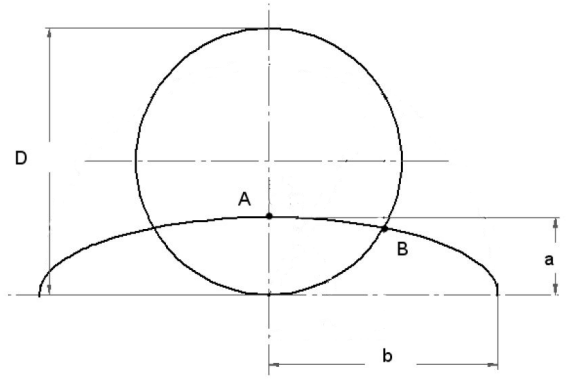

Figure 1: Geometry of the crack.

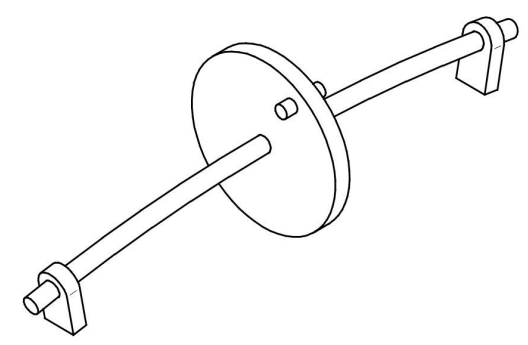

Figure 2: Jeffcott rotor with an eccentric mass.

To simulate the rotation of the shaft eight different angular positions, one for every eighth of a rotation has been considered, called angle of rotation $\phi$, see Figure 3. We analyze the static behavior of the shaft (considering the gravity effect) at each angular position. The influence of the mass eccentricity on the opening of the crack has been studied considering that the eccentric mass is placed on the disc and situated at different angles measured from the position of the crack, angle of eccentricity $\theta$, as shown in Figure 4. To simulate the effect of the eccentric mass, an inertial force, $F_{e}$ corresponding to a mass $m$ located at a distance $e$ from the center of the Jeffcott rotor rotating with the angular rotating velocity $\Omega$ has been included in the analysis, see Figure 5. Values taken in the analysis are $m=0.2 \mathrm{Kg}, e=80 \mathrm{~mm}$ and $\Omega=1000 \mathrm{rpm}$.
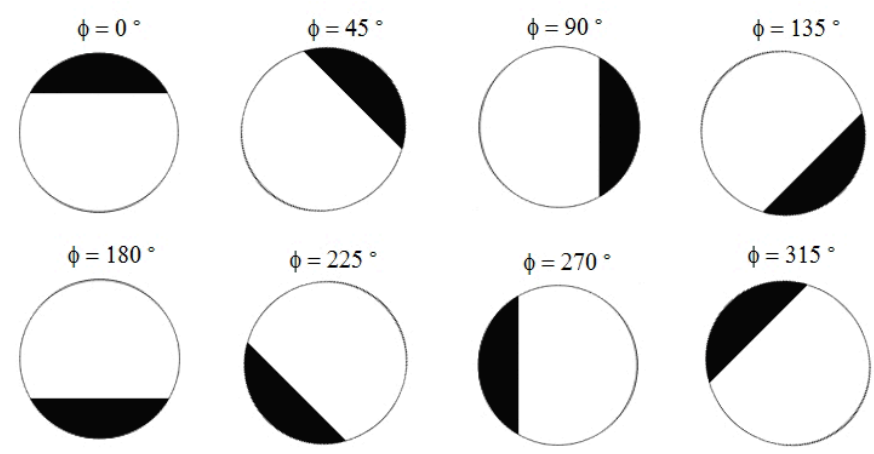

Figure 3: Angular positions of the crack during one rotation

Cracks of three lengths, $\alpha=a / D$, and different elliptical shapes (ellipticity ratio), $\beta=a / b$, have been modeled to evaluate the influence of both the crack size and the position of the eccentricity in the behavior of the shaft. The numerical simulation of the problem 


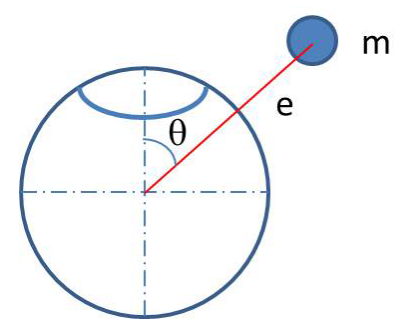

Figure 4: Relative position of the eccentricity

has been carried out using the Finite Element commercial code ABAQUS. For this analysis, the complete 3D model of the shaft has been considered due to there is no symmetry in the evaluated problem. The mesh of the three dimensional model is made employing 8 node linear brick elements. In order to avoid the interpenetration between the crack faces, a surface-tosurface contact interaction has been defined.

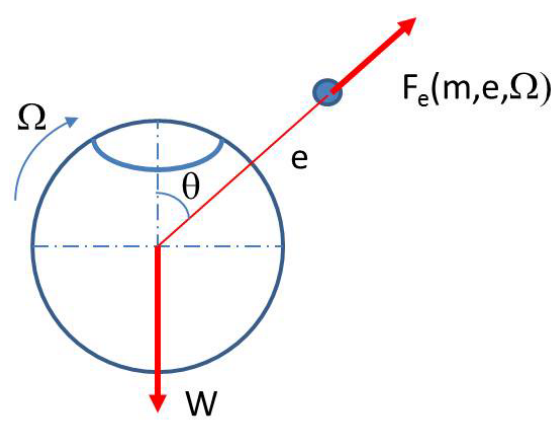

Figure 5: Resume of the applied forces on the shaft

A total of 256 cases have been simulated and analyzed accordingly with the following:

- no crack

- crack length $\alpha=0.1 ; 0.3 ; 0.5$

- crack shape $\beta=0 ; 0.25 ; 0.5 ; 0.75$

- eccentricity angle $\theta=0^{\circ} ; 45^{\circ} ; 90^{\circ} ; 135^{\circ} ; 180^{\circ}$

- rotation angle $\phi=0^{\circ} ; 45^{\circ} ; 90^{\circ} ; 135^{\circ} ; 180^{\circ} ; 225^{\circ} ; 270^{\circ} ; 315^{\circ} ; 360^{\circ}$

As metioned before three crack lengths have been analysed, but we have focused on some cases:

- for the smaller crack, $\alpha=0.1$, the four ellipticities have been considered as well as the eight angular positions and the five eccentricities. So, for the smaller crack, 160 cases has been simulated.

- for the medium crack, $\alpha=0.3$, two ellipticities, eight angular positions and one eccentricity corresponding to $90^{\circ}$, have been studied. 
- for the bigger crack $\alpha=0.5$, two ellipticities, eight angular positions and one eccentricity corresponding to $90^{\circ}$, have been studied.

The reason for this selection is based on the fact that it is very interesting to know the behaviour of the shaft with incipient and shallow cracks. On the other hand, in a previous study carried out by the authors [12] on the analysis of the breathing crack in shafts with cracks having straight front it was observed that a change in the behaviour of the unbalanced cracked shafts for values of eccentricity of $\theta=90^{\circ}$. The intention of this work is to evaluate if the same behaviour takes place for shafts with cracks having elliptical fronts.

To simulate the balanced shaft, 64 cases corresponding to the same three crack lengths and eight rotation angles have been also modeled in order to compare with the corresponding unbalanced cases.

\section{CRACK OPENING}

\subsection{Crack opening in a balanced shaft}

To analyse the breathing behaviour of a crack with a elliptical front during a rotation, a numerical simulation has been carried out. The study is focused on the opening of the cracked zone as the shaft rotates. An example of the results given by the simulations is in Figure 6. It shows the representation of the cracked section of a balanced shaft for a crack with $\alpha=0.5$, where the dark zone corresponds to the open part of the crack during a rotation. Each image represents the situation for each rotation angle.

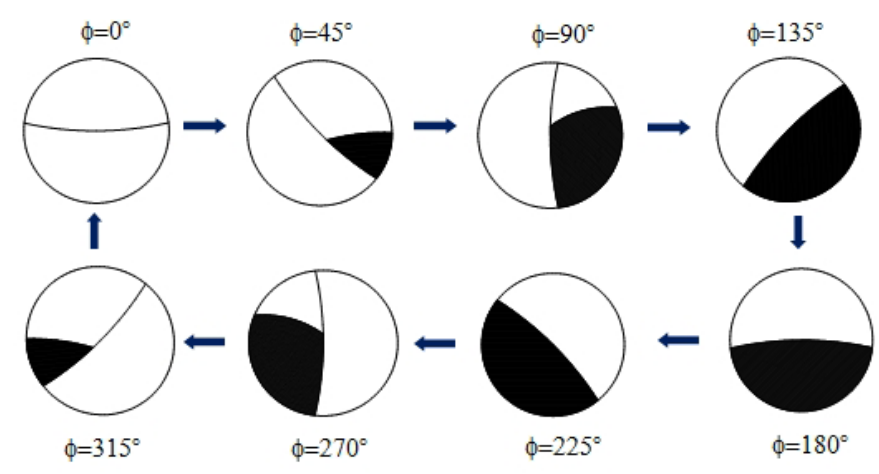

Figure 6: Opening of the crack (in black) for a balanced shaft with $\alpha=0.5$ and $\beta=0.5$ in a full rotation

From those images, the open area is very easy to detect and can be easily measured. In order to analyse and to compare the results obtained for the different cases, the percentage of opening area has been derived as (1):

$$
\Lambda=\frac{A_{o}}{A_{c}} \cdot 100
$$

where $A_{o}$ and $A_{c}$ are, respectively, the measured open area for the current case and the total cracked area. This means that $\Lambda$ will take values from 0 to 100 , being 0 for the case of a completely closed crack and 100 for fully open cracks. 


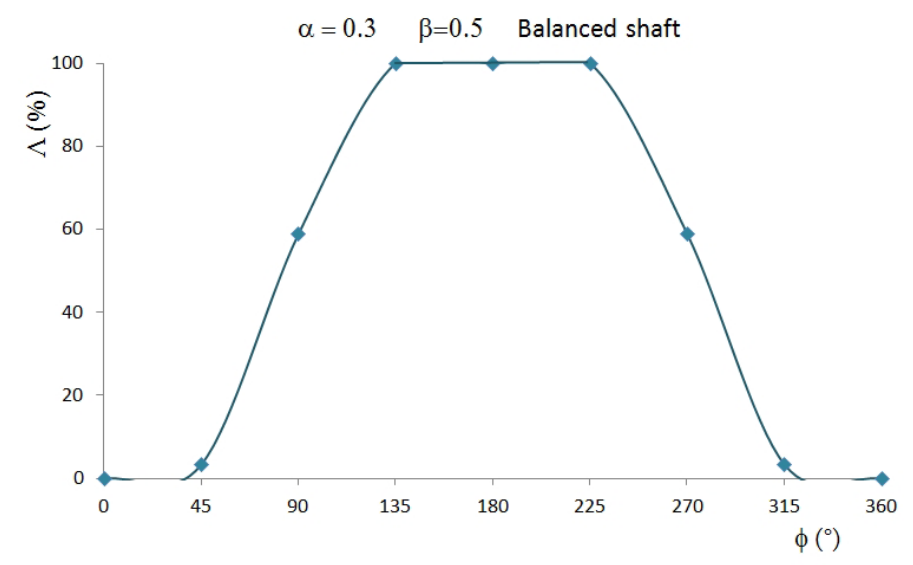

Figure 7: Opening of the crack in a rotation of the balanced shaft

The results of the percentage of opening area, $\Lambda$, for a balanced shaft are plotted first in Figure 7. Here, $\Lambda$ has been plotted versus rotation angle $\phi$ for the case of $\alpha=0.3$. Datum of Figure 7 correspond to those of Figure 6. This figure shows that while no eccentricity is introduced in the problem, the crack opens and closes as the shaft rotates with symmetry. In a whole rotation, the crack is fully closed for angles between $\phi=0^{\circ}$ to nearly $\phi=45^{\circ}$ and from nearly $\phi=315^{\circ}$ to $\phi=45^{\circ}$, that is for less than a quarter of a rotation. The crack is fully open for angles of rotation between $\phi=135^{\circ}$ to $\phi=225^{\circ}$, that is, for another quarter of rotation. So in half the rotation, the crack is partially open. In order to know how the breathing is for other crack lengths, a extension of this curve is shown in Figure 8. In this figure one can observe that for an angle of rotation, the opening of the crack is greater proportionally as the crack is longer. Another observation is that as the crack gets longer the part of the rotation in which the crack is open is greater, in fact a crack of $\alpha=0.5$ is always open except for the angle $\phi=0^{\circ}$. These curves will be taken as a reference to analyze the effect of the angle of the eccentricity.

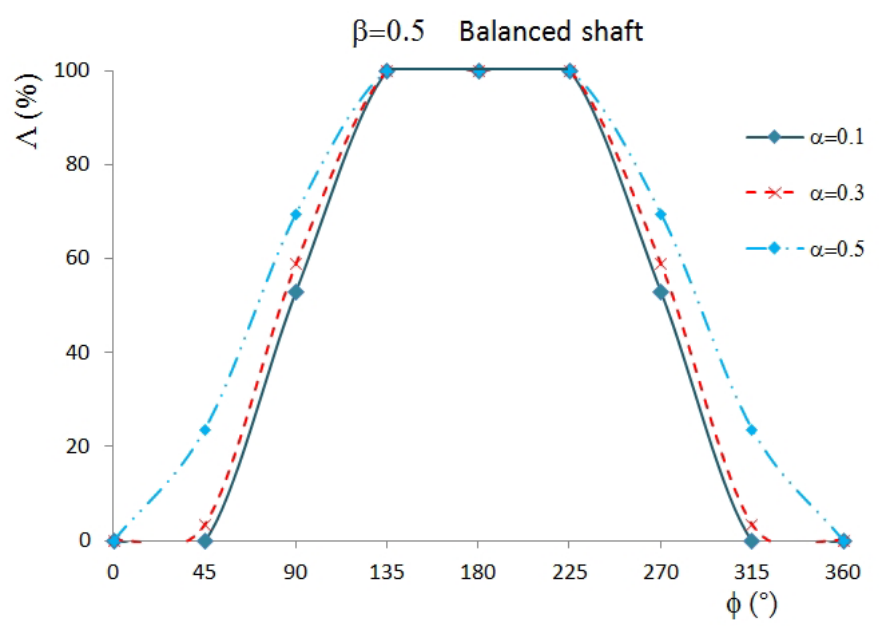

Figure 8: Opening of the crack in a balanced shaft in a rotation for different crack lengths 


\subsection{Crack opening of an unbalanced shaft}

When the eccentric mass is introduced in the problem, the effects can be observed in Figure 9. Here, the opening of the crack with $\alpha=0.3$ and $\beta=0.5$ is shown for different values of the rotation angle $\phi$ for the balanced shaft and for a shaft with an eccentricity at $\theta=90^{\circ}$. As can be seen, when a eccentric mass is considered in an angle of $\theta=90^{\circ}$ with respect to the crack position for a crack of medium length $(\alpha=0.3)$, the crack is never fully closed neither fully opened. It is always partially open and the percentage of opening is around half of the crack all over the rotation.

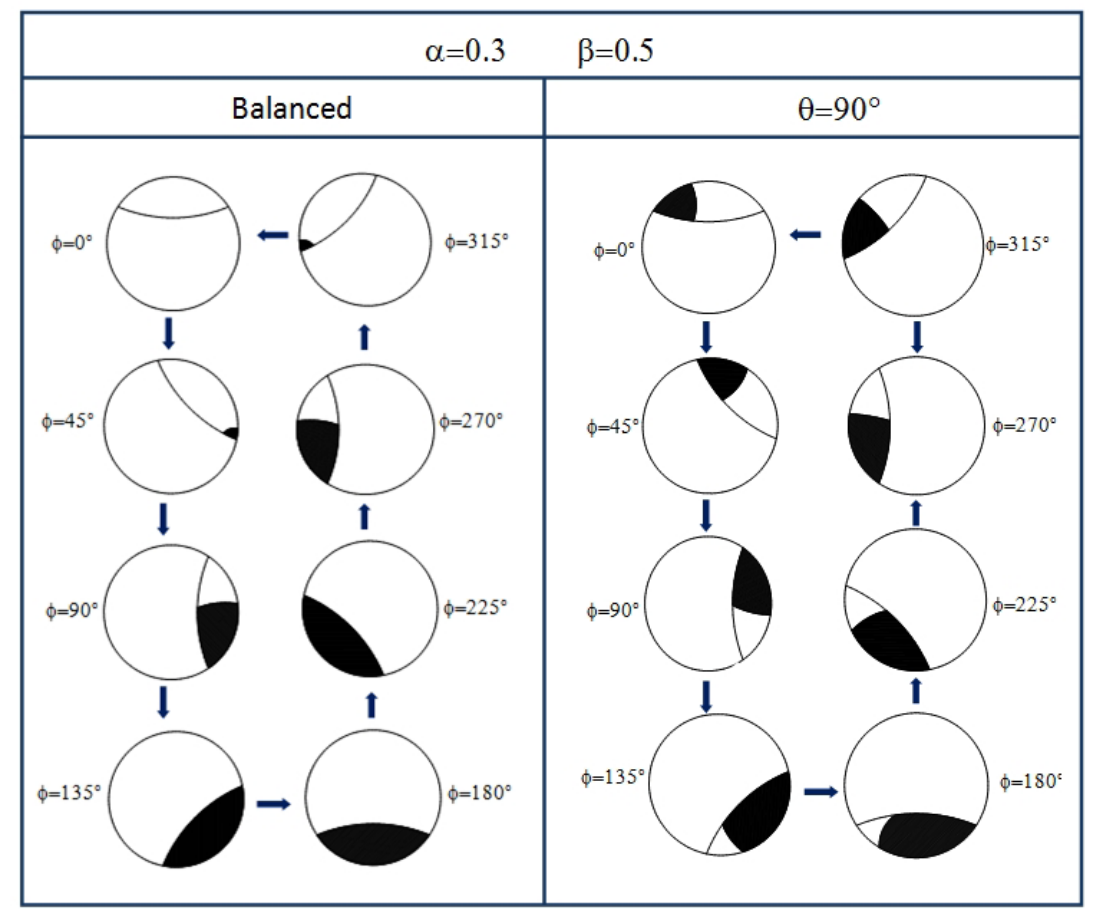

Figure 9: Opening of the crack (in black) for different angles of rotation, $\phi$. Case $\alpha=0.3$ and $\beta=0.5$

In order to study the effect of the position of the eccentric mass with respect to the crack, the opening of a crack of $\alpha=0.1$ has been analysed for a complete rotation and for different angles of eccentricity. In Figure 10 the proportional open area is plotted against the rotation angle for different eccentricity angles for the cases and for the four mentioned ellipticity ratio $\beta=0 ; 0.25 ; 0.5 ; 0.75$.

Looking to the figures we can see that, for the selected $m, e, \Omega$ and $\alpha$, if the eccentricity is placed opposite to the crack (eccentricity angle $\theta=180^{\circ}$ ), the crack never opens, which means that the cracked shaft behaves as it was an intact shaft. In this situation the crack will never propagate. If the eccentric mass is at the same position as the crack (eccentricity angle $\theta=0^{\circ}$ ) the crack will be always open. In this situation the crack will be in a good position to propagate. As the eccentric mass gets closer to the crack position (eccentricity angle changing from $180^{\circ}$ to $0^{\circ}$ ), the shaft passes from having the crack always closed to having the crack always open. When the eccentricity is located at $\theta=90^{\circ}$ the crack is partially open during the entire rotation for to the shortest ellipticities $(\beta=0$ and $\beta=0.25)$. As the ellipticities are greater the percentage of opening gets similar to that of the balanced shaft during the rotation. 


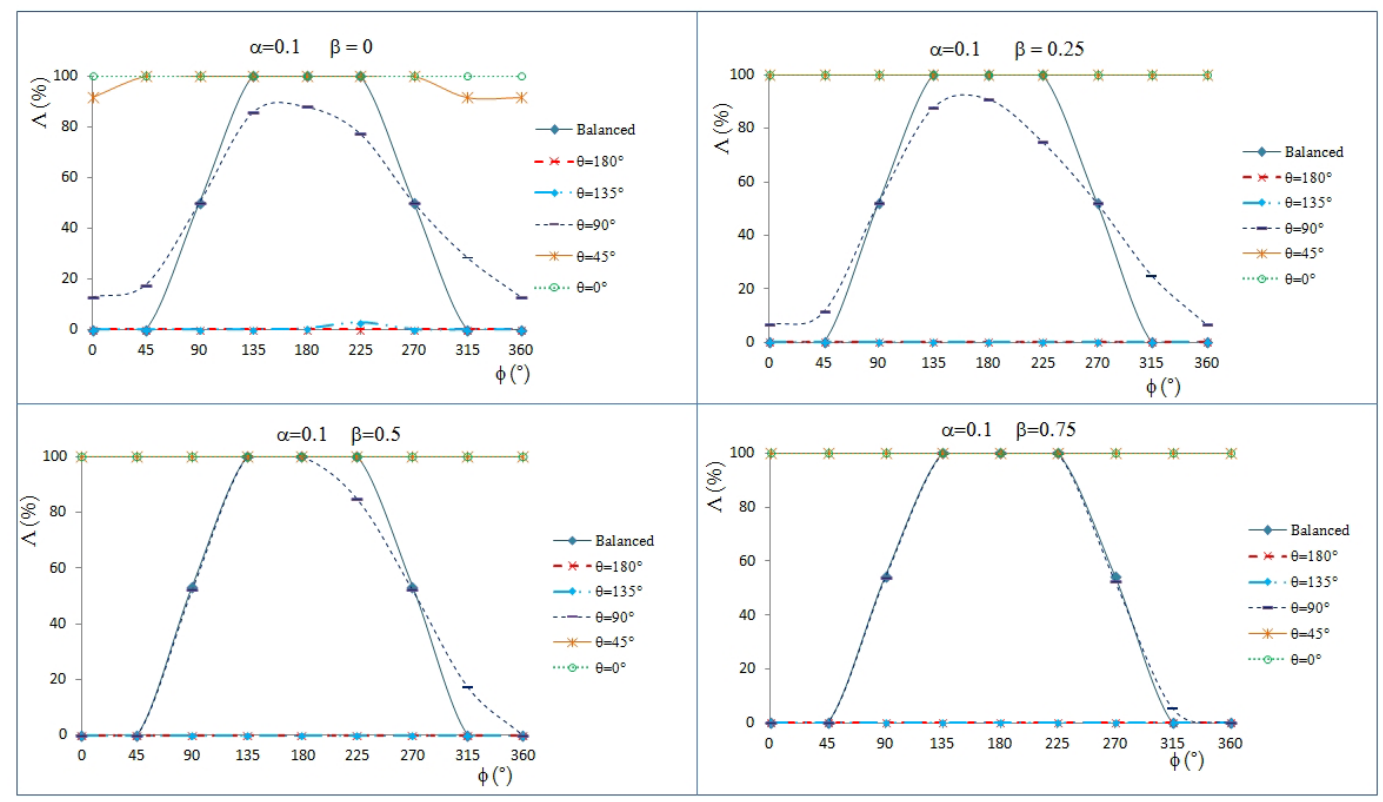

Figure 10: Opening of the crack in a rotation for different eccentricities and ellipticities. Case $\alpha=0.1$

A comparison of the opening of the crack for the location $\theta=90^{\circ}$ is shown in Figure 11. In this figure, the opening of the crack of $\alpha=0.1$ is plotted in a rotation for a the different values of the ellipticity. Looking to the curves, a change in the opening can be observed with the ellipticity. While the angle of rotation is between $\phi=270^{\circ}$ and $\phi=90^{\circ}$ the less the ellipticity, the more the opening of the crack. One the rotation passes through $\phi=90^{\circ}$ the situation reverses and the less the ellipticity, the less the opening of the crack. This behaviour is maintained up to $\phi=270^{\circ}$. It is also observed that the maximum of the opening is reached at lower angles of rotation as the ellipticity takes greater values.

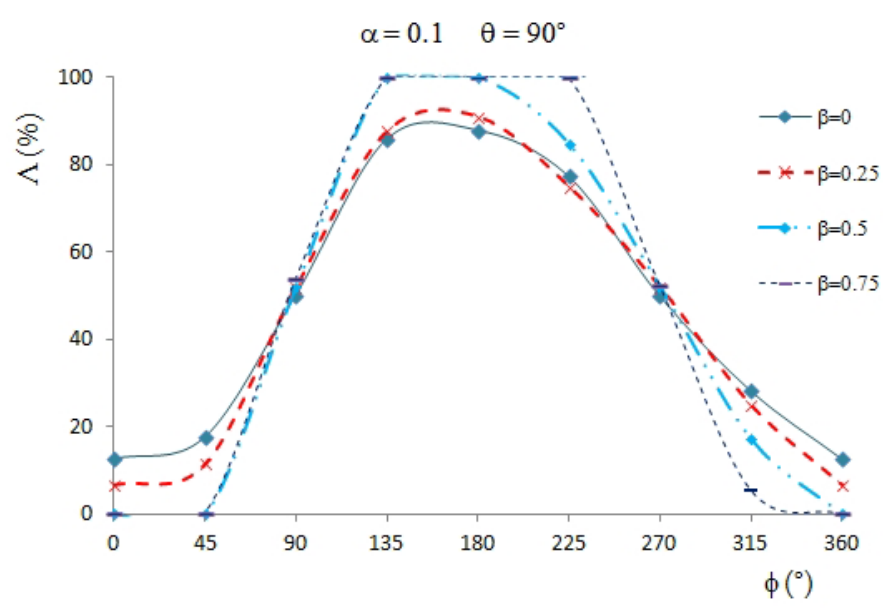

Figure 11: Opening of the crack in a rotation for $\alpha=0.1$ and $\theta=90^{\circ}$ for different ellipticities.

In Figure 13 a comparison of the opening of the cracks for the analysed eccentricity and for the different crack lengths is shown. As can be seen, the medium and large cracks have a similar behaviour showing curves of the same shape being greater, proportionally, the 
opening of the large crack. However, the shallow cracks $\alpha=0.1$ show a different opening pattern. This difference is due to we are comparing the relative open area and it looks like it is more difficult for a shallow crack to open and close, or more precisely, to change the current situation. The values of area of the open part of the crack with respect to the transversal area of the shaft in a rotation, just for illustration, are shown in Figure ??. Here, it can be seen that the open area grows with the length of the crack as it was expected.

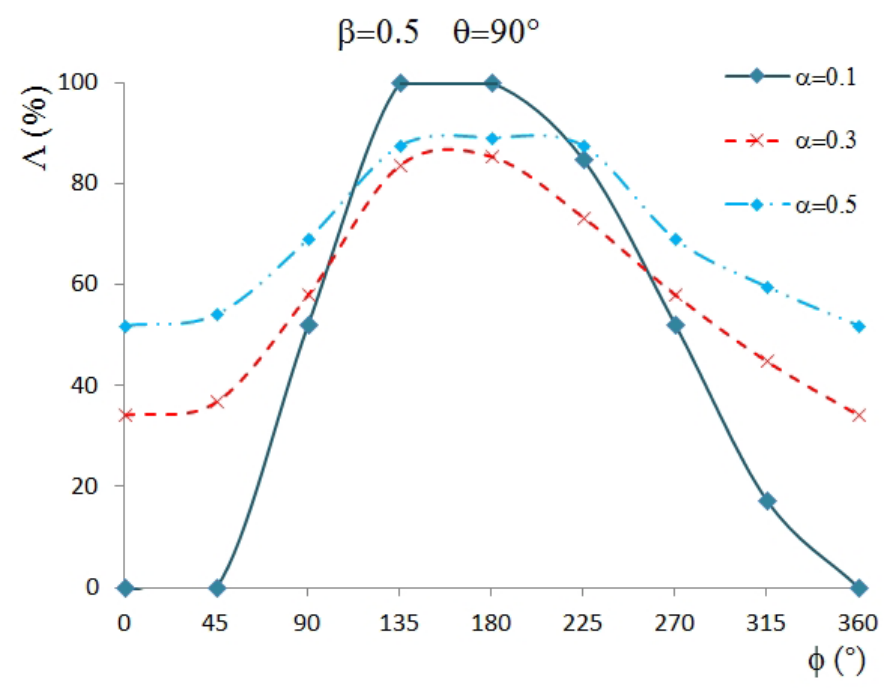

Figure 12: Opening of the crack in a rotation for $\theta=90^{\circ}$ for different crack lengths.

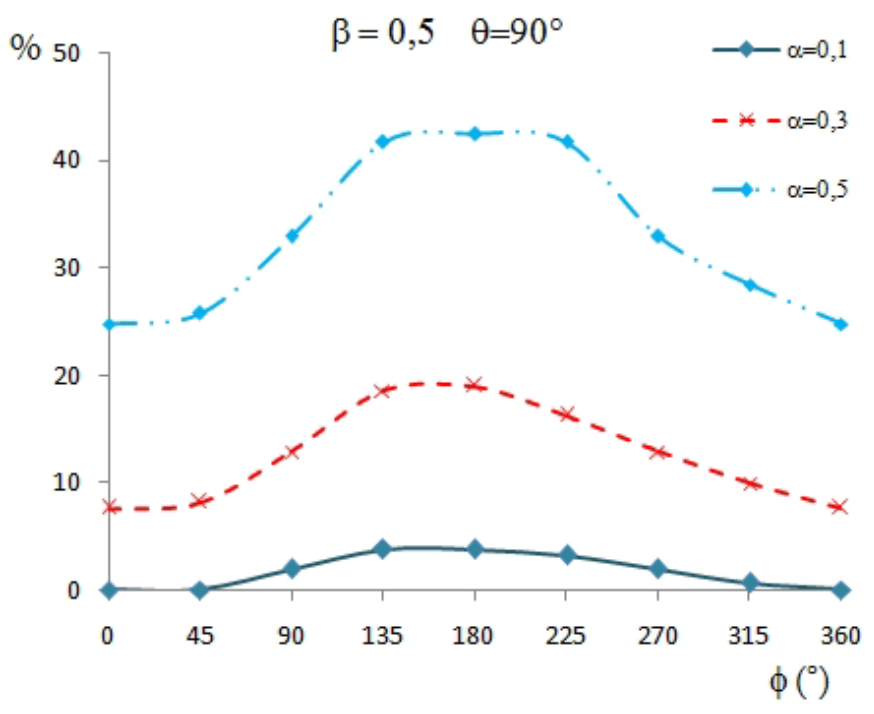

Figure 13: Absolute opening of the crack in a rotation for $\theta=90^{\circ}$ for different crack lengths.

\section{CONCLUSIONS}

As a conclusion we can resume that a numerical quasi-static simulation of the rotation of a cracked shaft containing an elliptical crack with a eccentric mass, that changes its 
position, has been carried out. From the numerical results, obtained for given values of the eccentricity set $m, e$, and for a given velocity $\Omega$, some basic ideas can be extracted:

- if the eccentricity is placed opposite to the crack and the crack is small, the crack never opens so that the crack would never propagate.

- if the eccentric mass is in front of the crack, the crack will always be open and very likely propagates.

- the percentage of opening increases with the length of the crack.

- for an eccentricity location of $\theta=90^{\circ}$ a change in the behaviour is produced when the shaft passes through the angle of rotation of $\theta=90^{\circ}$ and $\theta=270^{\circ}$. Between those angles, as the crack is more. elliptical there is more percentage of crack open for the same angle. Out of these angles the situation is just the opposite.

- for an eccentricity location of $\theta=90^{\circ}$ and for low values of ellipticity ( $\beta=0$ and $\beta=0.25$ ) the crack remains open during the whole revolution.

The work allows to know the influence of the unbalance of rotating shafts in the crack breathing mechanism and will allow to predict the influence of this behavior on the values of the stress intensity factor and in the propagation of the cracks.

\section{Acknowledgements}

The authors gratefully acknowledge the financial support given by the Spanish Ministerio de Economia y Competitividad, through the Project DPI2009-13264, for the development of this work. 


\section{REFERENCES}

[1] Bachschmid N., Pennacchi P., Tanzi E., "Some remarks on breathing mechanism, on nonlinear effects an on slant and helicoidal cracks". MSSP 22, 879-904, 2008.

[2] Carpinteri A., Brighenti R., Spagnoli A., "Surface flaws in cylindrical shafts under rotary bending". Fatigue Fract. Eng. Mat. Struct. 21, 1027-1035,1998.

[3] Cheng L., Li N., Chen X.F., He Z.J., "The influence of crack breathing and imbalance orientation angle on the characteristics of the critical speed of a cracked rotor". J.Sound Vib. 330, 2031-2048, 2011.

[4] Couroneau N., Royer J., "Simplified model for the fatigue growth analysis of surface cracks in round bars under mode I'. Int. J. Fatigue 20, 711-718, 1998.

[5] Darpe A.K., Gupta K., Chawla A., "Transient response and breathing behaviour of a cracked Jeffcott rotor". J.Sound Vib. 272, 207-243, 2004.

[6] Darpe A.K., Gupta K., Chawla A., "Dynamics of a bowed rotor with a transverse surface crack". J.Sound Vib. 296, 888-907, 2006.

[7] Darpe A.K. "A novel way to detect transverse surface crack in a rotating shaft". J.Sound Vib. 305, 151-171, 2007.

[8] Dimarogonas A.D., Papadopoulos C.A., "Vibration of cracked shafts in bending". J.Sound Vib. 91, 583-593, 1983.

[9] Jun O.S., Gadala M.S., "Dynamic behaviour analysis of a cracked rotor". J.Sound Vib. 309, 210-245, 2008.

[10] Patel T.H., Darpe A.K. "Influence of crack breathing model on nonlinear dynamics of a cracked rotor". J.Sound Vib. 311, 1953-1972, 2008.

[11] Penny J.E.T., Friswell M.I., "Simplified modelling of rotor cracks". Proc. of ISMA: Int. Conf. on Noise and Vibration engineering 2, 607-615, 2002.

[12] Rubio L., Muñoz-Abella B., Rubio P., Montero L., "Influence of the eccentricity in the crack breathing in a rotating shaft". Proc. 8th Int. Conf. Eng. Comp. Tech.ECT. Croatia, 2012.

[13] Rubio L., Muñoz-Abella B., Loaiza G., "Static behaviour of a shaft with an elliptical crack". Mech. Syst. Signal Pr. 25, 1674-1686, 2011.

[14] Sekhar A.S., Prabhu B.S. "Condition monitoring of cracked rotors through transient response". Mech. Mach. Theory 33, 1167-12751, 1998.

[15] Shin C.S., Cai C.Q., "Experimental and finite element analyses on stress intensity factors of an elliptical surface crack in a circular shaft under tension and bending". Int. J. Fracture 129, 239-264, 2004. 